\title{
Effect of deer density on tick infestation of rodents and the hazard of tick-borne encephalitis. II: Population and infection models
}

\author{
L. Bolzoni ${ }^{1, *}$, R. Rosà ${ }^{1}$, F. Cagnacci, A. Rizzoli \\ Department of Biodiversity and Molecular Ecology, Research and Innovation Centre - Fondazione Edmund Mach, San Michele all'Adige, Trento, Italy
}

\section{A R T I C L E I N F O}

\section{Article history:}

Received 10 November 2011

Received in revised form 1 February 2012

Accepted 16 February 2012

Available online 13 March 2012

\section{Keywords:}

Deer abundance

Rodent tick burden

Dilution effect

Ixodes ricinus

TBEV persistence

Tick borne encephalitis

Basic reproduction number

\begin{abstract}
A B S T R A C T
Tick-borne encephalitis is an emerging vector-borne zoonotic disease reported in several European and Asiatic countries with complex transmission routes that involve various vertebrate host species other than a tick vector. Understanding and quantifying the contribution of the different hosts involved in the TBE virus cycle is crucial in estimating the threshold conditions for virus emergence and spread. Some hosts, such as rodents, act both as feeding hosts for ticks and reservoirs of the infection. Other species, such as deer, provide important sources of blood for feeding ticks but they do not support TBE virus transmission, acting instead as dead-end (i.e., incompetent) hosts. Here, we introduce an eco-epidemiological model to explore the dynamics of tick populations and TBE virus infection in relation to the density of two key hosts. In particular, our aim is to validate and interpret in a robust theoretical framework the empirical findings regarding the effect of deer density on tick infestation on rodents and thus TBE virus occurrence from selected European foci. Model results show hump-shaped relationships between deer density and both feeding ticks on rodents and the basic reproduction number for TBE virus. This suggests that deer may act as tick amplifiers, but may also divert tick bites from competent hosts, thus diluting pathogen transmission. However, our model shows that the mechanism responsible for the dilution effect is more complex than the simple reduction of tick burden on competent hosts. Indeed, while the number of feeding ticks on rodents may increase with deer density, the proportion of blood meals on competent compared with incompetent hosts may decrease, triggering a decline in infection. As a consequence, using simply the number of ticks per rodent as a predictor of TBE transmission potential could be misleading if competent hosts share habitats with incompetent hosts.
\end{abstract}

(c) 2012 Published by Elsevier Ltd. on behalf of Australian Society for Parasitology Inc.

\section{Introduction}

Tick-borne infections are mostly caused by pathogens that are transmitted among several competent hosts by ticks that become infected following a blood meal. Among the zoonotic infections transmitted by Ixodes ricinus, tick-borne encephalitis, Lyme disease, rickettsiosis and anaplasmosis are emerging as international human health threats (Parola, 2004; Jensenius et al., 2006; Stanek, 2009).

Ticks belonging to the I. ricinus complex can feed on a wide range of host species, some of which are competent for the transmission of a given pathogen. In the case of Lyme disease in the north eastern USA, where the white-footed mouse is the main competent host, detailed studies have shown that the tick burden per mouse and the fraction of ticks infected with Borrelia burgdorferi is inversely correlated with the abundance of incompetent host species, such as the chipmunk, grey squirrel and white-tailed deer

\footnotetext{
* Corresponding author. Tel.: +39 0461 939545; fax: +39 0461948190.

E-mail address: luca.bolzoni@iasma.it (L. Bolzoni).

1 These authors contributed equally to this work.
}

(Ostfeld and Keesing, 2000; Schmid and Ostfeld, 2001; Ostfeld et al., 2006). This phenomenon is known as the dilution effect, since the presence of incompetent hosts diverts tick bites from competent hosts, reducing disease prevalence (Van Buskirk and Ostfeld, 1995; Norman et al., 1999). In this way, biodiversity loss is believed to increase pathogen transmission (Keesing et al., 2010). LoGiudice et al. (2003) pointed out that the dilution effect may not exclusively be an effect of species diversity, but could be a result of the positive correlation between diversity and the relative abundance of incompetent hosts in a community.

In the case of tick-borne encephalitis (TBE) in Europe, other host species are involved in transmission, but their functional role in TBE virus (TBEV) circulation is similar to the case of Lyme disease in the USA. Deer species (mainly roe and red deer) represent an important source of blood for all I. ricinus tick stages (e.g., Carpi et al., 2008; Kiffner et al., 2010), but are not competent for TBEV transmission, while wild rodents, which are responsible for much of the transmission, are generally hosts for the immature tick stages only (Perkins et al., 2003, 2006; Rizzoli et al., 2009). An empirical comparison among sites with contrasting TBEV occurrence in Italy and Slovakia has assessed the association between 
TBE hazard in the enzootic cycle and deer abundance (Cagnacci et al., 2012). Deer density has been shown to have an initial positive effect on the number of feeding ticks on rodents, to then reach a threshold value above which the effect becomes negative, as deer apparently divert ticks from feeding on rodents (Cagnacci et al., 2012). On the other hand, in the same study it was shown that the probability of TBEV occurrence in rodents and ticks is a monotonically decreasing function of deer density.

In this paper, we develop mathematical models that explore the dynamics of both tick populations and TBEV infection with the aim of interpreting the empirical results obtained by Cagnacci et al. (2012) in a robust theoretical framework. Specifically, the objective of this exercise is to understand the mechanisms that link the relative abundance of deer species (family Cervidae) to the tick population dynamics, in particular to the number of ticks feeding on rodents, which has been often used as a primary predictor of TBEV maintenance in the natural cycle (Randolph et al., 1996, 1999). In addition, the analysis of a TBEV infection model allows us to quantify, through computation of the basic reproduction number, the conditions that would lead to TBEV persistence, and to evaluate the effect of competent and incompetent host densities on the threshold of persistence. We use the basic reproduction number as a measure of TBEV transmission potential within the enzootic cycle, which represents (together with human exposure) a fundamental component of the overall risk of human infection (Randolph, 2010).

The ultimate purpose of this research is to compare model outputs with empirical results obtained from experiments carried out in Italy and Slovakia in order to explain the variation in tick abundance and TBEV occurrence observed at different experimental sites, and to provide a useful tool for identifying potential TBEV circulation foci. Finally, the infection model allows us to make helpful predictions of pathogen dynamics under different ecological scenarios.

Tick-borne infection systems incorporate interesting complexities as a result of a series of heterogeneities coupled with non-linear phenomena operating in the transmission processes between ticks, hosts and pathogens (Randolph et al., 2002a). The investigation of these complexities has required the development of several theoretical models. The first step has been the development of mathematical models for tick population dynamics by Sandberg et al. (1992), Kitron and Mannelli (1994), and Randolph and Rogers (1997), followed by the implementation of specific models for tickborne infections. Further models, based on ordinary differential equations (ODE), have been developed by several authors (see for instance Norman et al., 1999, 2004; Gilbert et al., 2001; Rosà et al., 2003; Rosà and Pugliese, 2007). One of the advantages in using ODE models is the possibility of estimating threshold conditions which permit a pathogen to persist, such as the basic reproduction number. However, despite their simplicity, these models usually require the estimation of several ecological and epidemiological parameter values. Basic models describe the tick population through a single dynamical variable (see Norman et al., 2004), while more realistic models include detailed information of the tick life cycle including tick life stages (larvae, nymphs and adults) with stage progression occurring through blood meals on different vertebrate hosts. For instance, two-vertebrate host systems including tick life stages have been developed in Norman et al. (1999) and Rosà et al. (2003), while a system with a three-host community has been investigated in Gilbert et al. (2001). In even more complex models, the explicit modelling of the different phases of tick activity has been introduced: specifically, the questing phase, where ticks seek a host on the vegetation, and the feeding phase, where ticks take their blood meal on the host (Rosà and Pugliese, 2007).
Other sources of complexity in the description of tick-borne infections arise from the different possible transmission pathways. One route of transmission takes place from an infected tick to a susceptible host and vice versa, often denoted as 'systemic transmission'. This route of transmission takes place via the host's blood circulatory system where a viraemia is produced; when the viraemia is sufficiently high, a biting tick will acquire the virus. An important advance in the understanding of how tick-borne pathogens persist in natural systems was the discovery of non-systemic transmission through co-feeding ticks taking place on some host species, such as rodents (Labuda et al., 1993a).

This route of transmission takes place via the host's skin, thereby avoiding the circulatory system, and viraemia is not needed. Specifically, non-systemic transmission occurs horizontally between ticks feeding together on the same host, and is considered crucial for the persistence of some infections, notably the TBEV complex (Randolph et al., 1996, 2002a). Trans-ovarial transmission, from adult ticks to offspring, is also known to occur, but its frequency is very low, and its contribution to TBEV transmission is generally thought to be negligible (Nuttall and Labuda, 2003).

\section{Materials and methods}

Here we consider simple deterministic models both for the dynamics of the tick I. ricinus and the transmission of the TBEV. The models consider two classes of hosts: wild rodents (named as $H_{R}$ ) and deer (named as $H_{D}$ ). Tick dynamics is based on the biology of I. ricinus which is the main vector for TBEV transmission. Ixodes ricinus has a life cycle that develops from the egg through two immature stages (larva and nymph) to the adult. Each immature stage requires a blood meal from a suitable vertebrate host. The adult female requires a meal before producing eggs once and then dying. Adult females can only obtain a feed from large mammals i.e., deer, whilst the immature stages will also feed on smaller warm-blooded vertebrates such as rodents.

We built a model that explicitly takes into account the different tick activity phases (i.e., questing and feeding on different hosts). Such a framework follows the empirical analyses by Cagnacci et al. (2012), where feeding ticks on rodents are related to deer abundance. On the other hand, we prefer to keep the model as simple as possible in order to provide general analytical results and, at the same time, to avoid hyper-parameterisation. Then, following Norman et al. (2004) and Porter et al. (2011), we added together all tick stages to obtain the equations for total tick dynamics. In addition, to suitably describe some biological processes that involve specific tick stages, such as the production of larvae (sustained only by adults) and non-systemic transmission (sustained only by nymphs), we assumed a fixed proportion of tick individuals within each developmental stage. Randolph (2004) showed that the latter assumption could be considered biologically acceptable as ixodid ticks display a remarkably constant population size and maintain a quite regular proportion of individuals within each developmental stage.

\subsection{Tick population dynamics}

Here we introduce the model for the dynamics of the tick vector I. ricinus. The model consists of three coupled differential equations describing the changes in the abundance of questing ticks, $T_{q}$, feeding ticks on rodents, $T_{f R}$, and feeding ticks on deer $T_{f D}$. The equations of the resulting model are as follows:

$$
\begin{aligned}
\frac{d T_{q}}{d t}= & b_{T R} a_{R} \sigma T_{f R}+b_{T D} a_{D} \sigma T_{f D}-d_{T} T_{q}-\left(\beta_{R} H_{R}+\beta_{D} H_{D}\right) T_{q}+(1 \\
& \left.-a_{R}\right) \sigma T_{f R}+\left(1-a_{D}\right) \sigma T_{f D}
\end{aligned}
$$


$\frac{d T_{f R}}{d t}=\beta_{R} H_{R} T_{q}-\sigma T_{f R}$

$\frac{d T_{f D}}{d t}=\beta_{D} H_{D} T_{q}-\sigma T_{f D}$

A full list of parameters with their biological interpretation is given in Table 1. Questing ticks die at rate $d_{T}$ and move into the feeding tick compartments by encountering hosts of either type $H_{R}$ and $H_{D}$ at rate $\beta_{R}$ and $\beta_{D}$, respectively. Once the blood meal is completed, ticks drop off their hosts at rate $\sigma$ (corresponding to an average duration of a blood meal of $1 / \sigma$ days). While dropped adult ticks (measured by the fraction $a_{i}$ ) reproduce at rate $b_{T i}$ and die, dropped immature stages $\left(1-a_{i}\right)$ return to the questing compartments (with $i=R, D$ ). We assume a density-dependent birth rate that can be written as $b_{T i}=r_{T} \exp \left(-s_{T} T_{f i}\right)$, where $r_{T}$ is the maximum egg production of adult ticks and $s_{T}$ is the strength of density-dependence.

\subsection{TBEV infection dynamics}

The model for TBEV infection is an extension of the model proposed by Norman et al. (2004) for tick-borne transmitted virus, deriving explicitly the equations for the activity phases (questing and feeding) of a tick population. The model consists of nine coupled differential equations describing the temporal variation in the abundance of ticks and hosts, subdivided into compartments with respect to the state of infection as in a classical SusceptibleInfected-Recovered (SIR) model. The first six equations describe the temporal variations of susceptible (questing, $T_{q}^{s}$, feeding on rodents, $T_{f R}^{s}$, and deer, $\left.T_{f D}^{s}\right)$ and infected ticks $\left(T_{q}^{i}, T_{f R}^{i}, T_{f D}^{i}\right)$. Empirical investigations on TBEV transmission have found that rodents are the most competent hosts (both for systemic and non-systemic transmission), while for deer no evidence of virus transmission has been observed (Labuda et al., 1993a). Hence, the last three equations describe the infection dynamics of susceptible $\left(H_{R}^{S}\right)$, infected $\left(H_{R}^{i}\right)$ and immune $\left(H_{R}^{r}\right)$ rodents while deer are assumed to be at constant density $\left(H_{D}\right)$. The equations of the resulting model are as follows:

$$
\begin{aligned}
\frac{d T_{q}^{s}}{d t}= & b_{T R} a_{R} \sigma T_{f R}^{s+i}+b_{T D} a_{D} \sigma T_{f D}^{s+i}-d T_{q}^{s}-\left(\beta_{R} H_{R}+\beta_{D} H_{D}\right) T_{q}^{s}+(1 \\
& \left.-a_{R}\right) \sigma T_{f R}^{s}+\left(1-a_{D}\right) \sigma T_{f D}^{s}
\end{aligned}
$$

$$
\begin{aligned}
\frac{d T_{q}^{i}}{d t}= & -d T_{q}^{i}-\left(\beta_{R} H_{R}+\beta_{D} H_{D}\right) T_{q}^{i}+\left(1-a_{R}\right) \sigma T_{f R}^{i}+\left(1-a_{D}\right) \sigma T_{f D}^{i} \\
\frac{d T_{f R}^{s}}{d t}= & (1-p) \exp \left[-n_{R} \theta T_{f R}^{i}\right] \beta_{R} H_{R}^{i} T_{q}^{s}+\exp \left[-n_{R} \theta T_{f R}^{i}\right] \beta_{R}\left(H_{R}^{s}+H_{R}^{r}\right) T_{q}^{s} \\
& -\sigma T_{f R}^{s} \\
\frac{d T_{f R}^{i}}{d t}= & \beta_{R} H_{R} T_{q}^{i}+p \beta_{R} H_{R}^{i} T_{q}^{s}+(1-p)\left(1-\exp \left[n_{R} \theta T_{f R}^{i}\right]\right) \beta_{R} H_{R}^{i} T_{q}^{s}+(1 \\
& \left.-\exp \left[n_{R} \theta T_{f R}^{i}\right]\right) \beta_{R}\left(H_{R}^{s}+H_{R}^{r}\right) T_{q}^{s}-\sigma T_{f R}^{i} \\
\frac{d T_{f D}^{s}}{d t}= & \beta_{D} H_{D} T_{q}^{s}-\sigma T_{f D}^{s} \\
\frac{d T_{f D}^{i}}{d t}= & \beta_{D} H_{D} T_{q}^{i}-\sigma T_{f D}^{i} \\
\frac{d H_{R}^{s}}{d t}= & b\left(H_{R}\right) H_{R}-d_{R} H_{R}^{s}-q \beta_{R} H_{R}^{s} T_{q}^{i} \\
\frac{d H_{R}^{i}}{d t}= & q \beta_{R} H_{R}^{s} T_{q}^{i}-\left(d_{R}+\gamma_{R}+\alpha_{R}\right) H_{R}^{i} \\
\frac{d H_{R}^{r}}{d t}= & \gamma_{R} H_{R}^{i}-d_{R} H_{R}^{r}
\end{aligned}
$$

A full list of parameters with their biological interpretation is given in Table 1 . Since TBEV infection may induce extra mortality $\left(\alpha_{R}\right.$, disease-related death rate) in some rodent species (Labuda et al., 1993b), we need to introduce its population dynamics into the model. Precisely, we define $d_{R}$ as the rodent natural mortality rate and $b\left(H_{R}\right)=b_{R} H_{R}-\left[\left(d_{R}-b_{R}\right) / K_{R}\right] H_{R}^{2}$, as its density-dependent fertility function, where $b_{R}$ represents competent host birth rate at low densities and $K_{R}$ represents its carrying capacity. Moreover, infected rodents may recover from TBEV infection at rate $\gamma_{R}$

Ticks feeding on infected competent hosts become infected, through the systemic transmission route, with probability $p$, while a competent host fed on by an infected tick has probability $q$ of becoming infected. Here, we assume that systemic infection occurs at the beginning of the blood meal, so that the infection rate of ticks [hosts] will be proportional to the encounter rate between

Table 1

Numerical values and biological interpretation of parameters used in the models.

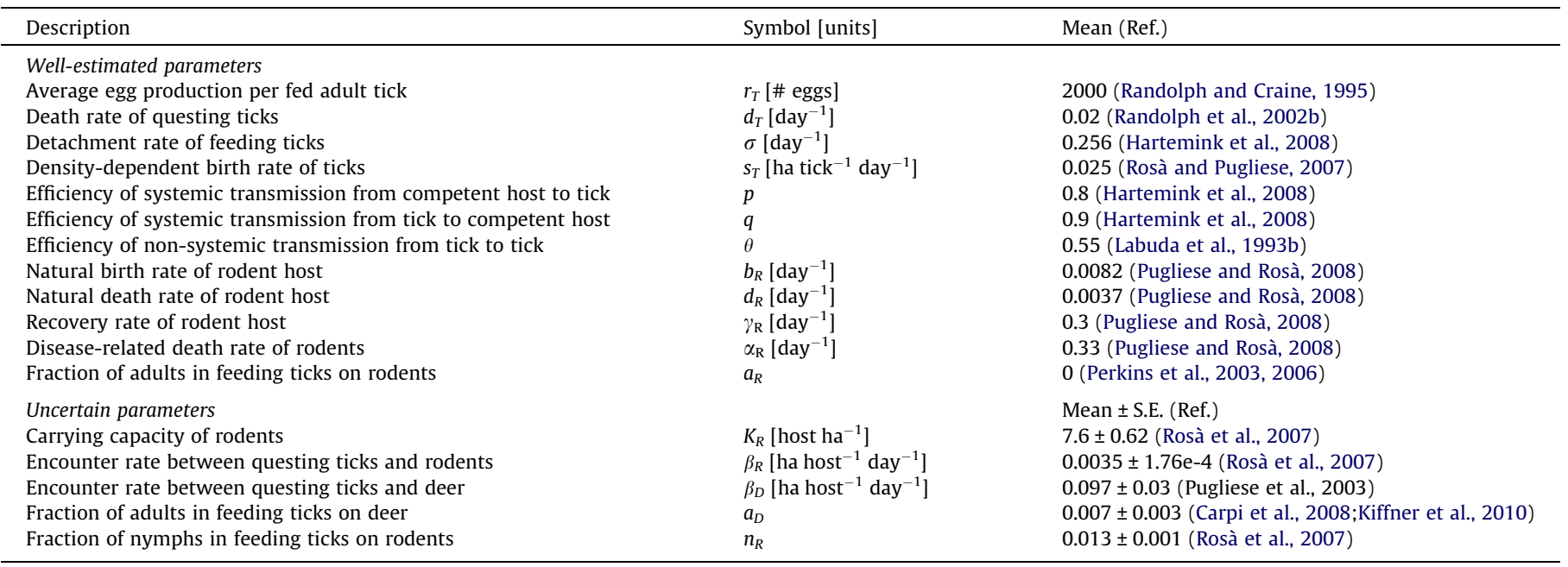


questing susceptible ticks $T_{q}^{s}$ [questing infected ticks $T_{q}^{i}$ ] and infected hosts $H_{R}^{i}$ [susceptible hosts $H_{R}^{s}$ ], i.e., $p \beta_{R} T_{q}^{s} H_{R}^{i}\left[q \beta_{R} T_{q}^{i} H_{R}^{s}\right.$.

As in Rosà and Pugliese (2007), we introduced a mechanism for non-systemic transmission (also knows as co-feeding) specific for TBEV. Precisely, we assumed the rate of non-systemic transmission to be proportional to the density of infected feeding ticks, $T_{f R}^{i}$, the density of susceptible questing ticks, $T_{q}^{s}$, and competent hosts, $H_{R}$ (precisely, $1-\exp \left[n_{R} \theta T_{f R}^{i}\right] \beta_{R} T_{q}^{S} H_{R}$ ). The proportion $n_{R} \theta$ describes the co-feeding transmission term, where $\theta$ is the probability of non-systemic transmission, and $n_{R}$ is the fraction of nymphs against the total number of ticks feeding on $H_{R}$ (since only nymphs may infect other ticks through co-feeding).

\subsection{Model parameter estimation}

Models (1) and (2) are calibrated with parameter values estimated from I. ricinus and TBEV infection, considering rodents and deer as the main hosts. Parameter values are summarised in Table 1 . While some of the parameters describing tick ecology and TBE epidemiology have been robustly estimated in the scientific literature, other parameter values - which are strongly related to the local ecological conditions, such as encounter rates $\left(\beta_{R}, \beta_{D}\right)$, rodent density $\left(H_{R}\right)$, and the fractions of ticks in different stages (i.e., $\left.n_{R}, a_{D}\right)$ - are more subject to site-specific variability. To overcome this uncertainty, we performed sensitivity analyses of model results assuming the uncertain parameters $\left(\beta_{R}, \beta_{D}, H_{R}, a_{D}\right.$, and $\left.n_{R}\right)$ as realisations from Gaussian distributions. To generate a plausible collection of parameter values, we used the Latin Hypercube Sampling method with 10,000 extractions (Hoare et al., 2008). Means and standard errors of the uncertain parameter distributions are derived from previous field studies, all conducted in the same study site in Trentino, northern Italy (Table 1 ). In particular, estimates of rodent density $\left(H_{R}\right)$, feeding ticks on rodents $\left(T_{f R}\right)$ and questing ticks $\left(T_{q}\right)$ are derived from a long-term study, where live-trapping rodent monitoring has been implemented using capture-mark-recapture technique, while the density of questing ticks was estimated from vegetation using a dragging technique (Rosà et al., 2007). Rodent density was estimated using a standard open population Jolly-Seber model (Krebs, 1989). For each rodent captured, a careful assessment of the number of different tick life stages feeding on that rodent was carried out (Rosà et al., 2007). These data also allow us to estimate the fraction of feeding ticks in each stage and, in particular, the fraction of nymphs feeding on rodents $\left(n_{R}\right)$.

Imposing $d T_{f R} / d t=0$ in model ( 1 ) and rearranging, we obtained the following relationship for calculating the encounter rate between ticks and rodents at the non-trivial equilibrium:

$\beta_{R}=\frac{T_{f R}}{H_{R}} \frac{\sigma}{T_{q}}$

Inserting in (3) the observed values of feeding ticks per rodent $\left(T_{f R} / H_{R}\right)$, questing ticks $\left(T_{q}\right)$ and the tick detachment rate $(\sigma)$ we obtained the mean and standard error of $\beta_{R}$ reported in Table 1.

No comparable measures existed for the same study area to estimate the encounter rates of ticks with deer $\left(\beta_{D}\right)$. However, an experiment with tracer animals (domesticated goats) was carried out at the same study site in Trentino (northern Italy), obtaining the numerical values reported in Pugliese et al. (2003) and summarised in Table 1.

Several empirical works performed in Trentino (northern Italy) show that wild rodents are generally hosts for the immature tick stages only, while adults can only obtain a suitable feed from large mammals (Perkins et al., 2003, 2006; Rizzoli et al., 2009). Thus, we assumed that the fraction of adult ticks feeding on rodents is negligible (i.e., we set $a_{R}=0$ ). In addition, Rosà and Pugliese (2007) showed that the assumption of neglecting the small fraction of
Table 2

Comparison between observed tick-borne encephalitis virus occurrence, column [TBE virus], and $R_{0}$ estimates in TBE virus-positive and TBE virus-negative sites, columns $\left[R_{0}^{\mathrm{TBE}}\right]$ and $\left[R_{0}^{\text {stand }}\right]$. Columns $\left[T_{f R} / H_{R}\right]$ and $\left[H_{D}\right]$ report the observed values of feeding ticks per rodent and deer density, respectively, used for $R_{0}$ computation as in Eq. (7) in Section 3.2, column $\left[R_{0}^{\mathrm{TBE}}\right]$. Columns $\left[L_{f R} / H_{R}\right]$ and $[\mathrm{Cof}]$ report the observed values of larval feeding ticks per rodents and number of co-feeding groups, respectively, used for standard $R_{0}$ computation as in Randolph et al. (1999), column $\left[R_{0}^{\text {stand }}\right]$. Data are from Cagnacci et al. (2012).

\begin{tabular}{|c|c|c|c|c|c|c|c|}
\hline $\begin{array}{l}\text { Site } \\
\text { (Country) }\end{array}$ & $\frac{T_{f R}}{H_{R}}$ & $H_{D}$ & $\frac{L_{f R}}{H_{R}}$ & Cof & $\begin{array}{l}\text { TBE } \\
\text { virus }\end{array}$ & $R_{0}^{\mathrm{TBE}} \pm \mathrm{S} . \mathrm{E}$. & $R_{0}^{\text {stand }}$ \\
\hline $\begin{array}{l}\text { Belluno } \\
\quad \text { (Italy) }\end{array}$ & 24.13 & 4.6 & 23.12 & 31.83 & Yes & $4.95 \pm 0.67$ & 7.52 \\
\hline $\begin{array}{l}\text { Grosotto } \\
\text { (Italy) }\end{array}$ & 1.09 & 6.8 & 1.09 & 0.00 & No & $0.71 \pm 0.18$ & 0.11 \\
\hline $\begin{array}{l}\text { Lamar } \\
\text { (Italy) }\end{array}$ & 16.93 & 2.2 & 16.17 & 21.14 & Yes & $3.80 \pm 0.54$ & 5.07 \\
\hline $\begin{array}{l}\text { Mazzo } \\
\quad \text { (Italy) }\end{array}$ & 0.53 & 3.1 & 0.42 & 0.43 & No & $0.49 \pm 0.074$ & 0.11 \\
\hline $\begin{array}{l}\text { Rozhanoce } \\
\quad \text { (Slovakia) }\end{array}$ & 1.63 & 15.5 & 1.60 & 8.3 & No & $0.89 \pm 0.10$ & 1.53 \\
\hline $\begin{array}{l}\text { Topolcianky } \\
\text { (Slovakia) }\end{array}$ & 4.40 & 2.0 & 3.90 & 1.00 & Yes & $1.60 \pm 0.48$ & 0.55 \\
\hline
\end{tabular}

adult ticks on rodents in the population model does not critically affect tick dynamics. Finally, the fraction of adult ticks feeding on deer $\left(a_{D}\right)$ was estimated using tick collection data from deer legs in Carpi et al. (2008). Since Kiffner et al. (2010) showed that the proportion of adult ticks feeding on legs is lower in comparison with the entire body, we corrected our estimate by assuming the legs-to-body tick ratio observed in Kiffner et al. (2010).

\subsection{Empirical assessment of the effect of deer on tick abundance and TBEV occurrence}

Following model selection results from Cagnacci et al. (2012) we selected the best generalised linear mixed-model (GLMM), with negative binomial error distribution, that included the effect of deer (linear and quadratic terms) and region on the number of feeding ticks on rodents (see Table 2 in Cagnacci et al., 2012). Then, to obtain a robust estimate of the observed value of deer density at which the peak of feeding ticks on rodents occurs $\left(\hat{H}_{D}^{\text {ticks }}\right)$, and to compute its standard error, we took into account the uncertainty in deer densities empirically detected at each study site (see Table 1 in Cagnacci et al., 2012). We assigned to deer density estimates a truncated Gaussian distribution representing the degree of uncertainty associated with empirical values. Specifically, by randomly extracting deer density values from a probability distribution constrained at $95 \%$ by the confidence intervals reported in Cagnacci et al. (2012) (see Table 1), we ran the best GLMM 1,000 times generating output distributions for each model coefficient (precisely: deer, $D_{1}$; deer ${ }^{2}, D_{2}$; and region, Region). In particular, from the GLMM coefficients related to deer we estimated the distribution of the deer density at which the peak of feeding ticks on rodents occurs $\left(\hat{H}_{D}^{\text {ticks }}\right)$ as $D_{1} /\left(2 * D_{2}\right)$.

In order to compare infection model prediction with empirical results on TBEV occurrence, we used the field estimates of the average number of larvae $\left(L_{f R} / H_{R}\right)$ and total tick $\left(T_{f R} / H_{R}\right)$ feedings on rodents, the number of co-feeding groups on rodents (Cof) and deer density $\left(H_{D}\right)$ in Cagnacci et al. (2012) to compute, through model (2), the expected basic reproduction number for TBEV $\left(R_{0}^{\mathrm{TBE}}\right)$ at each study site. The basic reproduction number measures the number of secondary cases produced by a single infection in a completely susceptible population. By definition, the pathogen can persist in the population when the mean number of infected hosts produced by a single infected host is larger than 1 $\left(R_{0}^{\mathrm{TBE}}>1\right)$. Finally, we compared the empirical results on TBEV 
occurrence with the expected $R_{0}$ computed through classical procedures for tick-borne infections (Randolph et al., 1999).

Statistical analyses were performed with statistical packages [R] 2.10.1 (2010, The R Foundation for Statistical Computing).

\section{Results}

\subsection{Tick population dynamics: model results and comparison with empirical data}

Before comparing model outputs with empirical results obtained from experiments described in Cagnacci et al. (2012), we briefly reviewed the main features of model (1). We considered tick dynamics in the absence of adult ticks feeding on rodents (i.e., $a_{R}=0$ ), since they generally feed on large mammals only (see Section 2.3 for details). Then, using next-generation matrix techniques (Diekmann et al., 1990), we computed for model (1) the following basic reproduction number of ticks:

$R_{0}^{\mathrm{ticks}}=\frac{r_{T} a_{D} \beta_{D} H_{D}}{\left(d_{T}+a_{D} \beta_{D} H_{D}\right)}$.

$R_{0}^{\text {ticks }}$ represents the expected number of ticks produced by a single tick, when density-dependent effects are absent, and defines the threshold for tick population to persist $\left(R_{0}^{\text {ticks }}>1\right)$. Similar to the model of Norman et al. (2004), Eq. (4) shows that $R_{0}^{\text {ticks }}$ in model $(1)$ is an increasing, saturating function of deer density $\left(H_{D}\right)$, and does not depend on rodent host density $\left(H_{R}\right)$.

From model (1), we analytically computed the mean number of feeding ticks per rodent host $\left(T_{f R} / H_{R}\right)$ at the non-trivial equilibrium, finding the following function of $H_{D}$ :

$\frac{T_{f R}}{H_{R}}=\frac{\beta_{R}}{S_{T} \beta_{D} H_{D}} \log \left(R_{0}^{\text {ticks }}\right)$.

In Fig. 1 we show how $T_{f R} / H_{R}$, computed as in (5), changes with deer density (solid curve). In particular, the solid curve is computed as the average of 10,000 extractions by using Latin Hypercube Sampling techniques from Gaussian distributions of the uncertain parameters with means and standard errors in Table 1, while the grey area represents the $95 \%$ confidence interval.

In the same figure we display the mean value of the empirical results obtained from the GLMM re-sampling procedure that allows for variability in deer densities estimates (dashed curve).

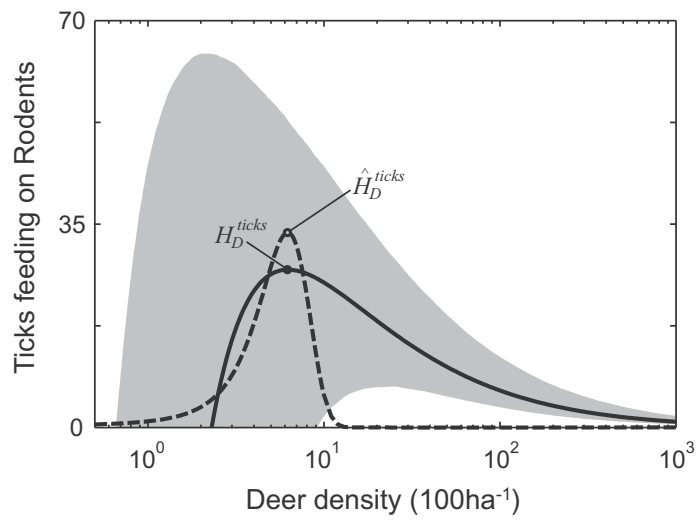

Fig. 1. Effect of deer density on the number of feeding ticks per rodent $\left(T_{f R} / H_{R}\right)$ as in Eq. (5) in Section 3.1 (solid curve) and from the empirical results obtained through the generalised linear mixed-model re-sampling procedure (dashed curve). The solid curve is obtained as the average of 10,000 extractions sampled from uncertain parameter distributions with means and standard errors as in Table 1, while the grey region represents the $95 \%$ confidence interval. Filled $\left(H_{D}^{\text {ticks }}\right)$ and open $\left(\hat{H}_{D}^{\text {ticks }}\right)$ circles represent the predictions by model (1) and the observed values of deer density at which the peak of the number of ticks feeding per rodent takes place. Other parameters are as in Table 1.
Theoretical and empirical results showed a qualitatively similar pattern of feeding ticks on rodents, reaching their peaks $\left(H_{D}^{\text {ticks }}\right.$ and $\hat{H}_{D}^{\text {ticks }}$, respectively) for intermediate levels of deer density.

Through algebraic manipulations of model (1), we computed the predicted value of deer density at which the peak of feeding ticks on rodent occurs $\left(H_{D}^{\text {ticks }}\right)$ at the non-trivial equilibrium, obtaining:

$H_{D}^{\mathrm{ticks}}=\frac{d_{T}}{\left(r_{T} / \tilde{R}_{0}^{\mathrm{ticks}}-1\right) a_{D} \beta_{D}}$

where $\tilde{R}_{0}^{\text {ticks }}$ is the solution of the implicit equation $R_{0}^{\text {ticks }} / r_{T}+\log \left(R_{0}^{\text {ticks }}\right)=1$ (see Proposition 1 in Supplementary data $\mathrm{S} 1$ for the proof). Since $R_{0}^{\text {ticks }}>1$ is a necessary condition for tick persistence, the solution of the previous expression satisfies the condition $\tilde{R}_{0}^{\text {ticks }}<r_{T}$ for every values of $r_{T}$. This implies that $H_{D}^{\text {ticks }}$ in (6) is always positive and, consequently, the relationship between deer and ticks on rodents is always hump-shaped for every combination of parameter values.

While values for adult tick egg production $\left(r_{T}\right)$ and death rate $\left(d_{T}\right)$ have been robustly estimated in several ecological surveys (Randolph and Craine, 1995; Randolph et al., 2002b), the fraction of adult ticks $\left(a_{D}\right)$ and the encounter rate between ticks and deer $\left(\beta_{D}\right)$, essential to quantify $H_{D}^{\text {ticks }}$, present a wide range of uncertainties. In order to evaluate the ranges of deer density values at which the peak of tick feeding on rodents occurs, a sensitivity analysis of these two parameters has been preformed and summarised in Fig. 2. Black dots represent $H_{D}^{\text {ticks }}$ values as in (6), where the pairs $\left(\beta_{D}, a_{D}\right)$ are sampled using Latin Hypercube Sampling techniques; Fig. 2 visualises the sampling along the $\beta_{D}$ axis (Fig. $2 \mathrm{~A}$ ) and $a_{D}$ axis (Fig. 2B). Black line $\bar{H}_{D}^{\text {ticks }}$ defines the average value of $H_{D}^{\text {ticks }}$ resulting from the $10,000\left(\beta_{D}, a_{D}\right)$ combinations, while dashed lines indicate the confidence interval of the observed peak estimated from the GLMM re-sampling procedure. Fig. 2 shows that the values predicted by model (1) of deer density at which the peak of feeding ticks occurs are in good agreement with empirical data. Indeed, the average value $\bar{H}_{D}^{\text {ticks }}=7.51$ host $100 \mathrm{ha}^{-1}$ (black line), falls within the confidence interval of the observed peak estimate $\left(4.8<\hat{H}_{D}^{\text {ticks }}<10.5\right.$ host 100 ha $\left.^{-1}\right)$.

\subsection{TBEV infection dynamics: model results and comparison with empirical data}

From TBEV infection model (2), we computed the basic reproduction number $\left(R_{0}^{\mathrm{TBE}}\right)$ with next-generation matrix technique (Diekmann et al., 1990) as the larger root of the following quadratic function (see Proposition 2 in Supplementary data S1 for details):

$R_{0}^{\mathrm{TBE}}=\max _{\lambda}\left(\operatorname{roots}\left(\lambda^{2}-\phi\left(H_{D}\right) \lambda-\varphi\left(H_{D}\right)=0\right)\right)$

where $\varphi\left(H_{D}\right)\left[\phi\left(H_{D}\right)\right]$ represents the contribution of infection spread from the systemic [non-systemic] transmission. By definition, the infection can persist in the population when the mean number of infected hosts produced by a single infected host, through both systemic and non-systemic transmission, is larger than $1\left(R_{0}^{\mathrm{TBE}}>1\right)$. From (7), we derive the threshold value for pathogen invasion as follows:

$$
\begin{aligned}
\varphi\left(H_{D}\right)+\phi\left(H_{D}\right)= & \frac{q p \beta_{R}^{2} T_{q} H_{R}}{\left(d_{R}+\gamma_{R}+\alpha_{R}\right)\left(d_{T}+a_{D} \beta_{D} H_{D}\right)} \\
& +\frac{n_{R} \theta \beta_{R} T_{q} H_{R}\left(d_{T}+\beta_{R} H_{R}+a_{D} \beta_{D} H_{D}\right)}{\sigma\left(d_{T}+a_{D} \beta_{D} H_{D}\right)} \\
= & 1 .
\end{aligned}
$$

Fig. 3 shows the region in the two-dimensional parameter space of deer $\left(H_{D}\right)$ and rodent $\left(H_{R}\right)$ densities where the basic reproduction number $R_{0}^{\mathrm{TBE}}$ is greater than 1 , taking into account the result of the 


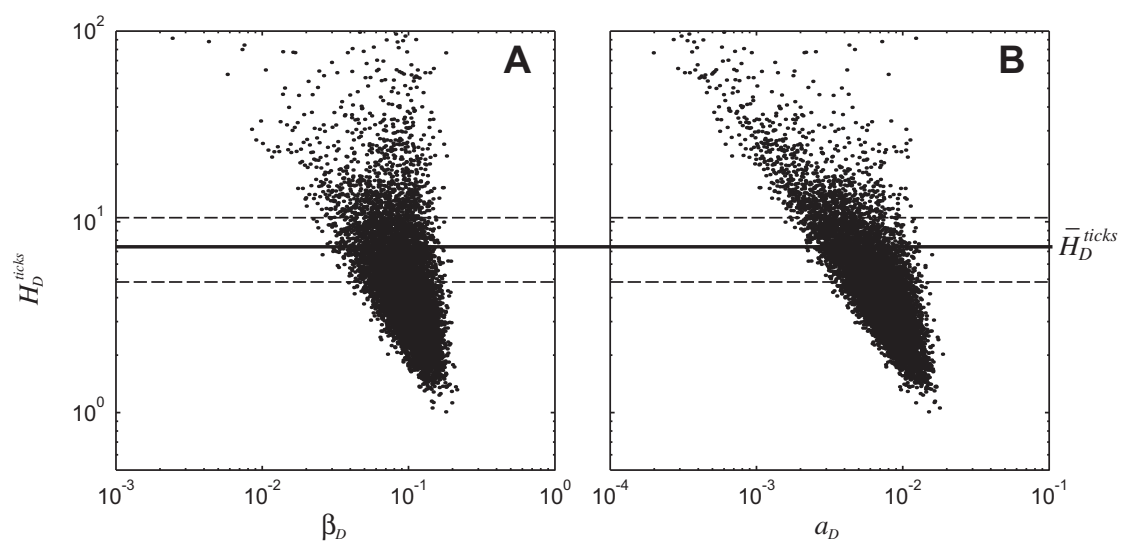

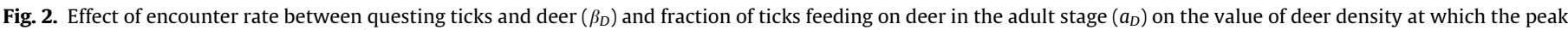

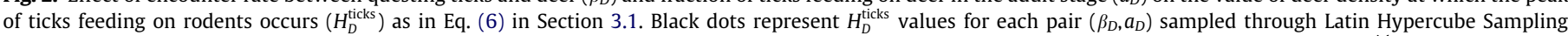

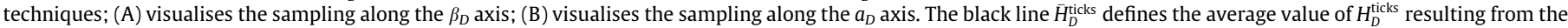

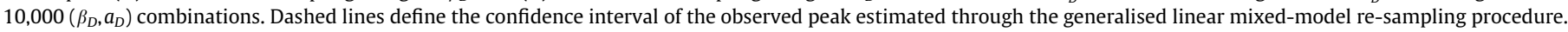
Other parameters are as in Table 1.

sensitivity analysis of uncertain parameters from Table 1 . The black curve represents the median value of $R_{0}^{\mathrm{TBE}}$ in 10,000 extractions, while the grey area boundaries represent the $95 \%$ confidence interval of the distribution.

We were interested to quantitatively validate model (2) results with data obtained empirically by Cagnacci et al. (2012) in six different TBE-negative and TBE-positive European locations. In Table 2, we provide model (2)'s estimated values of $R_{0}^{\mathrm{TBE}}$ at the six sampling sites by using mean values of field data for $T_{f R} / H_{R}$ and $H_{D}$ (see also Table 1 in Cagnacci et al., 2012), and using the parameter set, with their standard errors, as reported in Table 1 . These estimates were in very good agreement with observed data of presence and absence of TBEV recorded at the six locations (see columns [TBE virus] and $\left[R_{0}^{\mathrm{TBE}}\right]$ in Table 2 ). In addition, we showed that a standard procedure for $R_{0}$ computation, tailored by Randolph et al. (1999) for infection within Slovakian regions, may fail to predict TBEV presence/absence in some of our study sites (see column $\left[R_{0}^{\text {stand }}\right]$ in Table 2 ). More precisely, this procedure

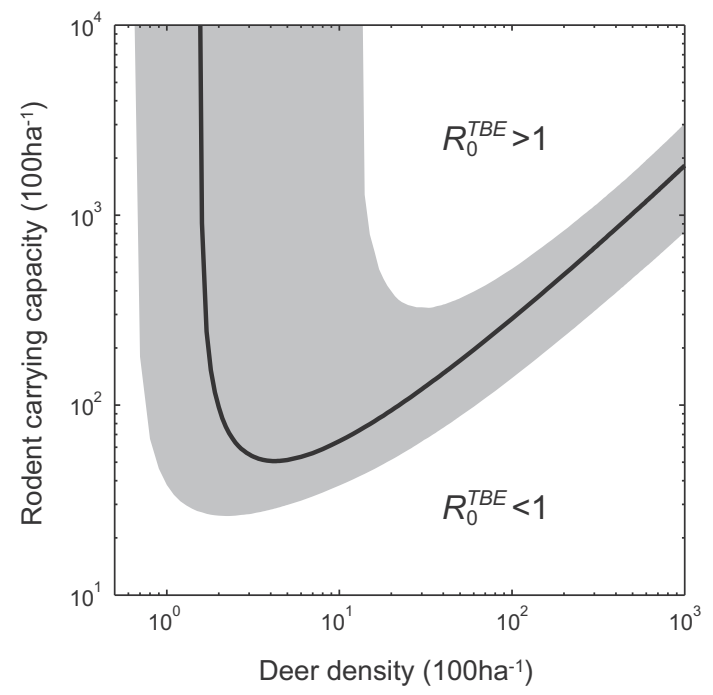

Fig. 3. Effect of deer density $\left(H_{D}\right)$ and rodent carrying capacity $\left(K_{R}\right)$ on $R_{0}^{\mathrm{TBE}}$ as in Eq. (7) in Section 3.2. The black curve is obtained as the median of 10,000 extractions sampled from uncertain parameter distributions with means and standard errors as in Table 1, while the grey region represents the 95\% confidence interval. Other parameters are as in Table 1. overestimates $R_{0}$ for sites with very high deer densities (as in Rozhanoce) since it does not take into account the deer dilution effect. On the other hand, it underestimates TBEV transmission potential where the low level of rodent tick burden (as in Topolcianky) is compensated by a deer density close to the value at which $R_{0}^{\mathrm{TBE}}$ is maximised.

In Fig. 4 we show the effect of deer density on the abundance of infected questing ticks ( $T_{q}^{i}$, grey curve) and competent hosts positive to TBEV ( $H_{R}^{i}+H_{R}^{r}$, black curve) as predicted by model (2). While the abundance of infected questing ticks displays a humpshaped behaviour that follows TBEV basic reproduction number values, as defined in (7), the abundance of positive rodents displays a more complex bi-modal relationship with deer density. This is due to the effect of the disease-related death $\left(\alpha_{R}\right)$ which depresses the total rodent population (and consequently its TBE-positive fraction) when TBEV is higher in ticks. Indeed, simulating model (2) in the absence of disease-related death, $\alpha_{R}=0$ (not shown here), we found a pattern in positive rodents similar to that showed for infected questing ticks.

Comparing expressions obtained through models (1) and (2), in particular (6) and (7), it is possible to analytically prove that the peak of TBEV basic reproduction number, $H_{D}^{\mathrm{TBE}}$, predicted by model (2) always occurs at a lower deer density than the peak for feeding ticks on rodents, $H_{D}^{\text {ticks }}$, predicted by model (1), which translates in the following mathematical inequality:

$$
H_{D}^{\mathrm{TBE}}=\arg \max _{H_{D}}\left[R_{0}^{\mathrm{TBE}}\right]<\arg \max _{H_{D}}\left[T_{f R} / H_{R}\right]=H_{D}^{\mathrm{ticks}}
$$

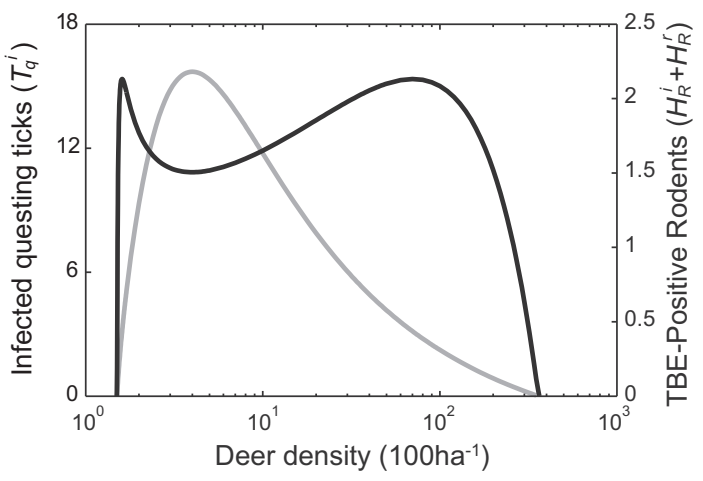

Fig. 4. Effect of deer density on the abundance of infected questing ticks ( $T_{q}^{i}$, grey curve) and the abundance of rodents positive to TBE virus $\left(H_{R}^{i}+H_{R}^{r}\right.$, black curve) as in model (2) in Section 2.2. Simulation parameters are as in Table 1. 
(see Proposition 3 in Supplementary data S1 for details). This implies that the number of secondary infections (measured by $R_{0}^{\mathrm{TBE}}$ ) may decline with deer density while the number of feeding ticks on rodents is still increasing.

\section{Discussion}

In this analysis, we investigated the contribution of incompetent hosts (such as deer) on tick vector abundance and on the persistence of TBEV. In particular, we were interested in providing a robust theoretical framework for the experimental results of Cagnacci et al. (2012) on the effect of deer density on TBEV transmission potential in some European countries, and to give insightful ecological interpretations of their results.

Tick life-cycle depends on the presence of large mammalian hosts for blood meals, and deer play a crucial role in the persistence of tick populations in different European regions (Rizzoli et al., 2009). On the other hand, deer are incompetent hosts for the transmission of TBEV, and their role in pathogen spread depends on whether the increase in vector density is sufficient to compensate for virus lost in 'wasted bites' on dead-end incompetent hosts (Hudson et al., 1995; Dobson and Foufopoulos, 2001).

In Italian and Slovakian study sites, Cagnacci and co-authors (2012) found a hump-shaped relationship between incompetent host density and the abundance of ticks feeding on competent hosts. They explained this result by suggesting that an initial increase in deer density amplifies the vector population by increasing the ratio of vector to susceptible rodent hosts, while a further increase 'dilutes' the number of ticks per rodents because deer at higher densities divert tick bites from them.

The analysis of the tick population model (1) agrees with the experimental findings, both qualitatively (Fig. 1) and quantitatively (Fig. 2), showing a peak of feeding ticks on competent hosts for intermediate densities of incompetent hosts. This pattern is the result of two competing effects driven by deer: on one hand, deer act as vector amplifiers; on the other hand, they also act as tick bite diverters from rodents. As deer are crucial for the tick life-cycle completion, an increase in their density will produce an increase in the total tick population. Initially, as the total number of ticks increases, we might expect more ticks feeding on rodents. However, when total ticks reach sufficiently high levels of abundance, density-dependent constraints would begin to take place, slowing down tick growth and saturating the tick population. Thus, a further increase in deer will have only a marginal effect on the increase in the tick population, while their effect on diverting bites from other hosts becomes more relevant, triggering the decrease of feeding ticks on rodents.

The exact nature of density-dependent regulation in ticks is not yet well understood. However, it is generally thought that the regulation is due to host immunity rather than direct competition or predation (Randolph, 2004). Indeed, Wikel (1996) and Brossard and Wikel (2004) showed that hosts acquire resistance to tick feeding as a result of repeated infestations. Their results are also supported by observations both in tick-cattle and tick-rodent interactions (Sutherst et al., 1979; Hughes and Randolph, 2001). Effects of acquired resistance in hosts have been observed, especially in tick fecundity, such as the increased duration of feeding, decreased number of eggs and reduced viability of those eggs (Bowessidjaou et al., 1977; Wikel, 1996; Brossard and Wikel, 2004). Hudson and Dobson (1995) showed that a decrease in the average blood meal might affect tick fecundity, as it varies directly with meal size. Here, we introduce the density-dependent regulation in our models, letting adult tick fecundity depend on the instantaneous total tick load, as proposed by Rosà and Pugliese (2007). The same authors also showed that the choice of shape of the density-dependent regulation function does not critically affect the conclusions for the role of deer on TBEV epidemiology (Rosà and Pugliese, 2007).

By analysing TBEV persistence through infection model (2), we found a pattern consistent with that of feeding ticks. In particular, we showed that the TBEV basic reproduction number, $R_{0}^{\mathrm{TBE}}$, is larger than one for intermediate levels of deer density (see Fig. 3) when the abundance of ticks on rodents is sufficient to sustain pathogen transmission, while for higher deer densities the infection dies out, supporting the 'wasted bites' hypothesis. This finding is in agreement with several theoretical results on tick-borne diseases, such as on TBEV in northern Italy (Rosà et al., 2003, 2007) and the louping ill virus system in upland Britain (Gilbert et al., 2001; Norman et al., 2004) showing this is not an artefact of this specific system. However, the comparison between tick population model (1) and TBEV infection model (2) outputs shows that the mechanism of 'wasted bites' seems more complex than a simple reduction of the number of feeding ticks on rodents. Indeed, we proved that the peak of TBEV basic reproduction number occurs at lower deer densities $\left(H_{D}^{\mathrm{TBE}}\right)$ than the peak of feeding ticks on rodents $\left(H_{D}^{\mathrm{ticks}}\right)$, highlighting that incompetent host density may depress the efficiency of pathogen transmission while still amplifying the tick burden on competent hosts. This result does not depend on the specific assumptions made in our models. Indeed, in more complex models where tick stage progression is included (see Rosà and Pugliese, 2007; Pugliese and Rosà, 2008), a similar pattern, with the peak of infection occurring at lower deer densities than the peak for feeding ticks on rodents, has been found. The biological explanation derives from the definition of basic reproduction number $\left(R_{0}^{\mathrm{TBE}}\right)$ as found in (7). Indeed, the peak of $R_{0}^{\mathrm{TBE}}$ occurs when the maximum number of ticks, which were previously infected on rodents, return to feed on rodents. Then, while the number of ticks on rodents may increase with deer density, as a consequence of the amplification of total tick population, the ratio of blood meals on competent hosts with respect to those on incompetent hosts may decrease, triggering an infection decline. Thus, using an estimation of the number of ticks per rodent as a predictor for TBEV transmission potential could be misleading, while taking into account the deer host density could increase the reliability of this prediction (see also Rizzoli et al., 2009). We showed that including the presence of incompetent deer hosts in the computation of the basic reproduction number is crucial to assess TBEV persistence at different sites, while simpler techniques based only on the tick burden on rodents may fail to assess it accurately (see Table 2 and Fig. 3). However, an in-depth host blood meal identification in questing ticks through molecular methods should provide a precise estimate of the ratio of blood meals on competent with respect to incompetent hosts that would represent a better proxy for the assessment of TBE transmission potential in the enzootic cycle.

Moreover, the results that emerged from the comparison between models ( 1 ) and (2) can also provide a possible explanation for the differences observed by Cagnacci et al. (2012) in the effect of deer density on the abundance in ticks feeding on rodents and TBEV occurrence. Cagnacci et al. (2012) found a hump-shaped relationship between feeding ticks on rodents and deer density, while they observed a monotonical decrease of TBEV occurrence probability with deer density. This discrepancy may be due to the deer density estimates obtained in their empirical assessment study. While the range of the estimated deer densities is well-centred to cover both the increasing and decreasing phases of the relationship between deer and feeding ticks on rodents, the same range of data could be not centred to catch the increasing phase of the relationship between deer and TBEV occurrence that model (2) predicts to occur at lower deer densities; as a consequence, only the decreasing phase is observed.

Model (2) could also explain the inconsistency in TBEV infection between ticks and rodents found by Cagnacci et al. (2012) (see 
Table 1). As shown in Fig. 4, the pattern of infection in rodents can differ dramatically from those in ticks, with the latter mimicking the behaviour of the basic reproduction number and the former shaped by the effect of the disease-related deaths. Labuda et al. (1993b) showed that disease-related death is strongly species-specific and can be high for certain rodent species (e.g., Pitymys subterraneus) whereas other species (e.g., Apodemus flavicollis) may develop low viraemias and are much more likely to survive infection. This result can have substantial consequences on TBE surveillance, where rodents (which are simpler to test) have been frequently used as sentinel animals (Hayasaka et al., 1999; Achazi et al., 2011), while pathogen persistence and the overall risk for human transmission seems better represented by the infection in ticks.

In the case of other tick-borne diseases, the role of tick hosts may be different from the TBE case. For instance, the lizard Sceloporus occidentalis, acts as an incompetent reservoir for Lyme disease (such as deer in the TBE system), while acting as a blood meal host for immature tick stages only (such as rodents in the TBE system). Swei et al. (2011) found experimentally that removing incompetent lizards may reduce the overall tick density and, thus, decrease disease risk.

Here we used deterministic models with constant coefficients, disregarding seasonality, a crucial feature in tick population dynamics in temperate regions (Randolph et al., 2002b). In the specific case of TBEV, the synchronisation of the annual tick cycle with those of competent rodents is one of the key factors for infection persistence (Randolph et al., 1999). However, the aim of this work was to highlight the mechanisms linking TBEV transmission to incompetent host density, so adding seasonal details would have obscured our main goal. Interestingly, Ghosh and Pugliese (2004) found a dilution effect triggered by incompetent host densities even in a discrete growing season model. In addition, seasonal models for vector-borne (Ogden et al., 2007; Nonaka et al., 2010), as well as directly transmitted diseases in wildlife (Bolzoni et al., 2008; Duke-Sylvester et al., 2011), are considerably more complex to investigate, but their predictions may allow us to understand intra- and inter-annual variability in disease infections in the near future.

Finally, the evaluation of the relative abundance of the key hosts involved in the pathogen transmission and the degree of host diversity within TBEV endemic areas is essential for a better evaluation of TBE risk. These issues, in particular the functional role of biodiversity, will be our focus in further investigations.

\section{Acknowledgements}

We are very grateful to Heidi Hauffe and Andrea Pugliese for their invaluable suggestions and comments that improved the manuscript. The authors thank two anonymous reviewers and the editor Ian Beveridge for providing observations and suggestions that led to a much improved version of the paper. L.B. was funded by the Autonomous Province of Trento (Italy), ACE-SAP project (regulation number 23, June 12th 2008, of the University and Scientific Research Service). This study was also partially funded by European Union (EU) Grant FP7-261504 EDENext and is catalogued by the EDENext Steering Committee as EDENext023 (http://www.edenext.eu). The contents of this publication are the sole responsibility of the authors and do not necessarily reflect the views of the European Commission.

\section{Appendix A. Supplementary data}

Supplementary data associated with this article can be found, in the online version, at http://dx.doi.org/10.1016/j.ijpara.2012. 02.006 .

\section{References}

Achazi, K., Ruzek, D., Donoso-Mantke, O., et al., 2011. Rodents as sentinels for the prevalence of tick-borne encephalitis virus. Vector-Borne Zoonotic Dis. 11, 641 647.

Bolzoni, L., De Leo, G.A., Gatto, M., Dobson, A., 2008. The effect of seasonality on epidemics of rabies in wildlife. Am. Nat. 172, 818-828.

Bowessidjaou, J., Brossard, M., Aeschlimann, A., 1997. Effect and duration of resistance acquires by rabbits on feeding and egg laying in Ixodes ricinus L.. Experientia 33, 528-530.

Brossard, M., Wikel, S.K., 2004. Tick immunobiology. Parasitology 129, S161-S176.

Cagnacci, F., Bolzoni L., Rosà, R., Carpi, G., Hauffe, H.C., Valent, M., Tagliapietra, V., Kazimirova, M., Koci, J., Stanko, M., Lukan, M., Henttonen, H., Rizzoli A., 2012. Effect of Deer density on tick infestation of rodents and the hazard of tick-borne encephalitis. Part I: empirical assessment. Int. J. Parasitol., this issue.

Carpi, G., Cagnacci, F., Neteler, M., Rizzoli, A., 2008. Tick infestation on roe deer in relation to geographic and remotely sensed climatic variables in a tick-borne encephalitis endemic area. Epidemiol. Infect. 136, 1416-1424.

Diekmann, O., Heesterbeek, J.A.P., Metz, J.A.J., 1990. On the definition and the computation of the basic reproduction ratio $R_{0}$ in models for infectious diseases in heterogeneous populations. J. Math. Biol. 28, 365.

Dobson, A., Foufopoulos, J., 2001. Emerging infectious pathogens of wildlife. Philos Trans. R. Soc. Lond. B 356, 1001-1012.

Duke-Sylvester, S.M., Bolzoni, L., Real, L.A., 2011. Strong seasonality produces spatial asynchrony in the outbreak of infectious diseases. J. R. Soc. Interface 8, 817-825.

Gilbert, L., Norman, R., Laurenson, K.M., Reid, H.W., Hudson, P.J., 2001. Disease persistence and apparent competition in a three-host community: an empirical and analytical study of large-scale, wild populations. J. Anim. Ecol. 70, 10531061 .

Ghosh, M., Pugliese, A., 2004. Seasonal population dynamics of ticks, and its influence on infection transmission: a semi-discrete approach. B. Math. Biol. 66, 1659-1684.

Hartemink, N.A., Randolph, S.E., Davis, S.A., Heesterbeek, J.A.P., 2008. The basic reproduction number for complex disease systems: defining $R_{0}$ for tick-borne infections. Am. Nat. 171, 743-754.

Hayasaka, D., Suzuki, Y., Kariwa, H., et al., 1999. Phylogenetic and virulence analysis of tick-borne encephalitis viruses from Japan and far-eastern Russia. J. Gen. Virol. 80, 3127-3135.

Hoare, A., Regan, D.G., Wilson, D.P., 2008. Sampling and sensitivity analyses tools (SaSAT) for computational modelling. Theor. Biol. Med. Model. 5, 4.

Hudson, P.J., Norman, R., Laurensona, M.K., Newborn, D., Gaunt, M., Jones, L., Reid, H., Gould, E., Bowers, R., Dobson, A., 1995. Persistence and transmission of tickborne viruses: Ixodes ricinus and louping-ill virus in red grouse populations. Parasitology 111, S49-S58.

Hudson, P.J., Dobson, A.P., 1995. Macroparasite: observed patterns. In: Grenfell, B.T. Dobson, A.P. (Eds.), Ecology of Infectious Diseases in Natural Populations. Cambridge University Press, Cambridge, UK, pp. 144-176.

Hughes, V.L., Randolph, S.E., 2001. Testosterone depress innate and acquired resistance to ticks in natural rodents hosts: a force for aggregated distributions of parasites. J. Parasitol. 87, 49-54.

Jensenius, M., Parola, P., Raoult, D., 2006. Threats to international travellers posed by tick-borne diseases. Travel Med. Infect. Dis. 4, 4-13.

Keesing, F., Belden, L.K., Daszak, P., Dobson, A., Harvell, C.D., Holt, R.D., Hudson, P., Jolles, A., Jones, K.E., Mitchell, C.E., Myers, S.S., Bogich, T., Ostfeld, R.S., 2010 Impacts of biodiversity on the emergence and transmission of infectious diseases. Nature 468, 647-652.

Kiffner, C., Lödige, C., Alings, M., Vor, T., Rühe, F., 2010. Abundance estimation of Ixodes ticks (Acari: Ixodidae) on roe deer (Capreolus capreolus). Exp. Appl. Acarol. 52, 73-84.

Kitron, U., Mannelli, A., 1994. Modeling the ecological dynamics of tick-borne zoonoses. In: Mather, T.N., Sonenshine, D.E. (Eds.), Ecological Dynamics of Tickborne Zoonoses. Oxford University Press, Oxford, UK, pp. 198-239.

Krebs, C.J., 1989. Ecological Methodology. Harper and Row, Publishers, New York, USA.

Labuda, M., Jones, L.D., Williams, T., Danielova, V., Nuttall, P.A., 1993a. Efficient transmission of tick-borne encephalitis virus between co-feeding ticks. J. Med. Entomol. 30, 295-299.

Labuda, M., Nuttall, P.A., Kozuch, O., Elecková, E., Williams, T., Zuffová, E., Sabó, A., 1993b. Non-viraemic transmission of tick-borne encephalitis virus: a mechanism for arbovirus survival in nature. Experientia 49, 802-805.

LoGiudice, K., Ostfeld, R.S., Schmidt, K.A., Keesing, F., 2003. The ecology of infectious disease: effects of host diversity and community composition on Lyme disease risk. Proc. Natl. Acad. Sci. USA 100, 567-571.

Nonaka, E., Ebel, G.D., Wearing, H.J., 2010. Persistence of pathogens with short infectious periods in seasonal tick populations: the relative importance of three transmission routes. PLoS One 5, e11745.

Norman, R., Bowers, B.G., Begon, M., Hudson, P.J., 1999. Persistence of tick-borne virus in the presence of multiple host species: tick reservoirs and parasite mediated competition. J. Theor. Biol. 200, 111-118.

Norman, R., Ross, D., Laurenson, M.K., Hudson, P.J., 2004. The role of non-viraemic transmission on the persistence and dynamics of a tick borne virus - Louping ill in red grouse (Lagopus lagopus scoticus) and mountain hares (Lepus timidus). J. Math. Biol. 48, 119-134.

Nuttall, P.A., Labuda, M., 2003. Dynamics of infection in tick vectors and at the tickhost interface. Adv. Virus Res. 60, 233. 
L. Bolzoni et al./International Journal for Parasitology 42 (2012) 373-381

381

Ogden, N.H., Bigras-Poulin, M., O’Callaghan, C.J., Barker, J.M., Kurtenbach, K., Lindsay, L.R., Charron, D.F., 2007. Vector seasonality, host infection dynamics and fitness of pathogens transmitted by the tick Ixodes scapularis. Parasitology 134, 209-227.

Ostfeld, R.S., Keesing, F., 2000. Biodiversity and disease risk: the case of Lyme disease. Conserv. Biol. 14, 722-728.

Ostfeld, R.S., Keesing, F., LoGiudice, K., 2006. Community ecology meets epidemiology: the case of Lyme disease. In: Collinge, S., Ray, C. (Eds.), Disease Ecology: Community Structure and Pathogen Dynamics. Oxford University Press, Oxford, UK, pp. 28-40.

Parola, P., 2004. Tick-borne rickettsial diseases: emerging risks in Europe. Comparative Immunology. Microbiol. Infect. Dis. 27, 297-304.

Perkins, S.E., Cattadori, I.M., Tagliapietra, V., Rizzoli, A., Hudson, P.J., 2003. Empirical evidence for key hosts in persistence of a tick-borne disease. Int. J. Parasitol. 33, 909-917.

Perkins, S.E., Cattadori, I.M., Tagliapietra, V., Rizzoli, A., Hudson, P.J., 2006. Localized deer absence leads to tick amplification. Ecology 87, 1981-1986.

Porter, R., Norman, R., Gilbert, L., 2011. Controlling tick-borne diseases through domestic animal management: a theoretical approach. Theor. Ecol. 4, 321-339.

Pugliese, A., Rosà, R., Ghosh, M., 2003. A mathematical model for tick-borne infections: a numerical study. In: Capasso, V. (Ed.), Mathematical Modelling \& Computing in Biology and Medicine. Esculapio, Bologna, Italy, pp. 464-469.

Pugliese, A., Rosà, R., 2008. Effect of host populations on the intensity of ticks and the prevalence of tick-borne pathogens: how to interpret the results of deer exclosure experiments. Parasitology 135, 1531-1544.

Randolph, S.E., Craine, N.G., 1995. General framework for comparative quantitative studies on transmission of tick-borne diseases using Lyme borreliosis in Europe as an example. J. Med. Entomol. 32, 765-777.

Randolph, S.E., Gern, L., Nuttal, P.A., 1996. Co-feeding ticks: epidemiological significance for tick-borne pathogen transmission. Parasitol. Today 12, 472479.

Randolph, S.E., Rogers, D.J., 1997. A generic population model for the African tick Rhipicephalus appendiculatus. Parasitology 115, 265-279.

Randolph, S.E., Miklisova, D., Lysy, J., Rogers, D.J., Labuda, M., 1999. Incidence from coincidence: patterns of tick infestations on rodents facilitate transmission of tick-borne encephalitis virus. Parasitology 118, 177-186.

Randolph, S.E., Chemini, C., Furlanello, C., Genchi, C., Hails, R.A., Hudson, P.J., Jones, L.D., Medley, G., Norman, R., Rizzoli, A., Smith, G., Woolhouse, M.E.J., 2002a. The ecology of tick-borne infections in wildlife reservoirs. In: Hudson, P.J., Rizzoli, A., Grenfell, B.T., Hesterbeek, H., Dobson, A.P. (Eds.), The Ecology of Wildlife Diseases. Oxford University Press, Oxford, UK, pp. 119-138.

Randolph, S.E., Green, R.M., Hoodless, A.N., Peacey, M.F., 2002b. An empirical quantitative framework for the seasonal population dynamics of the tick Ixodes ricinus. Int. J. Parasitol. 32, 979-989.

Randolph, S.E., 2004. Tick ecology: processes and patterns behind the epidemiological risk posed by ixodid ticks as vectors. Parasitology 129, S37S65.

Randolph, S.E., on behalf of the EDEN-TBD sub-project team, 2010. Human activities predominate in determining changing incidence of tick-borne encephalitis in Europe. Euro Surveillance 15, pii=19606.

Rizzoli, A., Hauffe, H.C., Tagliapietra, V., Neteler, M., Rosà, R., 2009. Forest structure and roe deer abundance predict tick-borne encephalitis risk in Italy. PLoS One 4 (2), e4336.

Rosà, R., Pugliese, A., Norman, R., Hudson, P.J., 2003. Thresholds for disease persistence in models for tick-borne infections including non-viraemic transmission, extended feeding and tick aggregation. J. Theor. Biol. 224, 359376.

Rosà, R., Pugliese, A., 2007. Effects of tick population dynamics and host densities on the persistence of tick-borne infections. Math. Biosci. 208, 216-240.

Rosà, R., Pugliese, A., Ghosh, M., Perkins, S.E., Rizzoli, A.P., 2007. Temporal variation of Ixodes ricinus intensity on the rodent host Apodemus flavicollis in relation to local climate and host dynamics. Vector Borne Zoonotic Dis. 7, 285-295.

Sandberg, S., Awerbuch, T.E., Spielman, A., 1992. A comprehensive multiple matrix model for tick Lyme disease. J. Theor. Biol. 157, 203-220.

Schmid, K.A., Ostfeld, R.S., 2001. Biodiversity and the dilution effect in disease ecology. Ecology 82, 609-619.

Stanek, G., 2009. Pandora's Box: pathogens in Ixodes ricinus ticks in Central Europe. Wien. Klin. Wochenschr. 121, 673-683.

Sutherst, R.W., Utech, K.B.W., Kerr, J.D., Whatron, R.H., 1979. Density-dependent mortality of the tick Boophilus microplus, on cattle-further observations. J. Appl. Ecol. 16, 397-403.

Swei, A., Ostfeld, R.S., Lane, R.S., Briggs, C.J., 2011. Impact of the experimental removal of lizards on Lyme disease risk. Proc. R. Soc. B 278, 2970-2978.

Van Buskirk, J., Ostfeld, R.S., 1995. Controlling Lyme disease by modifying the density and species composition of tick hosts. Ecol. Appl. 5, 1133-1140.

Wikel, S.K., 1996. Host immunity to ticks. Annu. Rev. Entomol. 41, 1-22. 